\title{
Why do we transition from walking to running? - Energy cost and lower leg muscle activity before and after gait transition under body weight support
}

\author{
Daijiro Abe ${ }^{\text {Corresp., } 1}{ }^{,}$Yoshiyuki Fukuoka ${ }^{2}$, Masahiro Horiuchi ${ }^{3}$ \\ ${ }^{1}$ Center for Health and Sports Science, Kyushu Sangyo University, Fukuoka, Japan \\ 2 Faculty of Health and Sports Science, Doshisha University, Kyotanabe, Kyoto, Japan \\ 3 Division of Human Environmental Science, Mt. Fuji Research Institute, Fujiyoshida, Yamanashi, Japan \\ Corresponding Author: Daijiro Abe \\ Email address: abed@ip.kyusan-u.ac.jp
}

Background. Minimization of the energetic cost of transport (COT) has been suggested for the walk-run transition in human locomotion. More recent literature argues that lower leg muscle activities are the potential triggers of the walk-run transition. We examined both metabolic and muscular aspects for explaining walk-run transition under body weight support (BWS; supported $30 \%$ of body weight) and normal walking (NW), because the BWS can reduce both leg muscle activity and metabolic rate.

Methods. Thirteen healthy young males participated in this study. The energetically optimal transition speed (EOTS) was determined as the intersection between linear CoT and speed relationship in running and quadratic CoT-speed relationship in walking under BWS and NW conditions. Preferred transition speed (PTS) was determined during constant acceleration protocol (velocity ramp protocol at 0.00463 $\mathrm{m} \cdot \sec ^{-2}\left(1 \mathrm{~km} \cdot \mathrm{h}^{-1}\right.$ per minute) starting from $1.11 \mathrm{~m} \cdot \mathrm{s}^{-1}$. Muscle activities and mean power frequency (MPF) were measured using electromyography of the primary ankle dorsiflexor (tibialis anterior; TA) and synergetic plantar flexors (calf muscles including soleus) before and after the walk-run transition.

Results. The EOTS was significantly faster than the PTS under both conditions, and both were faster under BWS than in NW. In both conditions, MPF decreased after the walk-run transition in the dorsiflexor and the combined plantar flexor activities, especially the soleus.

Discussion. The walk-run transition is not triggered solely by the minimization of whole-body energy expenditure. Walk-run transition is associated with reduced TA and soleus activities with evidence of greater slow twitch fiber recruitment, perhaps to avoid early onset of localized muscle fatigue. 


\section{Why do we transition from walking to running? -}

2 Energetic cost and lower leg muscle activity before and

3 after gait transition under body weight support

Daijiro Abe ${ }^{1}$, Yoshiyuki Fukuoka ${ }^{2}$, Masahiro Horiuchi ${ }^{3}$

${ }^{1}$ Center for Health and Sports Science, Kyushu Sangyo University, Fukuoka, Japan

${ }^{2}$ Faculty of Health and Sports Science, Doshisha University, Kyotanabe, Japan

${ }^{3}$ Division of Human Environmental Science, Mt. Fuji Research Institute, Fujiyoshida, Japan

Corresponding Author:

Daijiro Abe ${ }^{1}$

2-3-1 Matsukadai, Higashi-ku, Fukuoka City, Fukuoka 813-8503, Japan

Email address: abed@ip.kyusan-u.ac.jp

\section{Abstract}

Background. Minimization of the energetic cost of transport (CoT) has been suggested for the walk-run transition in human locomotion. More recent literature argues that lower leg muscle activities are the potential triggers of the walk-run transition. We examined both metabolic and muscular aspects for explaining walk-run transition under body weight support (BWS; supported $30 \%$ of body weight) and normal walking (NW), because the BWS can reduce both leg muscle activity and metabolic rate.

Methods. Thirteen healthy young males participated in this study. The energetically optimal transition speed (EOTS) was determined as the intersection between linear CoT and speed relationship in running and quadratic CoT-speed relationship in walking under BWS and NW conditions. Preferred transition speed (PTS) was determined during constant acceleration protocol (velocity ramp protocol at $0.00463 \mathrm{~m} \cdot \mathrm{sec}^{-2}\left(1 \mathrm{~km} \cdot \mathrm{h}^{-1}\right.$ per minute) starting from $1.11 \mathrm{~m} \cdot \mathrm{s}^{-}$

1. Muscle activities and mean power frequency (MPF) were measured using electromyography of the primary ankle dorsiflexor (tibialis anterior; TA) and synergetic plantar flexors (calf muscles including soleus) before and after the walk-run transition.

Results. The EOTS was significantly faster than the PTS under both conditions, and both were faster under BWS than in NW. In both conditions, MPF decreased after the walk-run transition in the dorsiflexor and the combined plantar flexor activities, especially the soleus. 
34 Discussion. The walk-run transition is not triggered solely by the minimization of whole-body energy expenditure. Walk-run transition is associated with reduced TA and soleus activities with evidence of greater slow twitch fiber recruitment, perhaps to avoid early onset of localized muscle fatigue.

\section{Introduction}

Human terrestrial locomotion involves two major gait patterns: walking and running. A biological benefit of these two patterns is in facilitation of economical energy expenditure (Margaria, 1938). When humans gradually increase their walking speed, they reach a speed at which they naturally change their gait pattern from walking to running, and it has been suggested that such a walk-run gait transition minimizes their metabolic rate (Cavagna \& Kaneko, 1977; Minetti AE, Ardigò LP, Saibene, 1994). In walking, there is a U-shaped relationship between the energetic cost of transport per unit distance (CoT) and speed (s); for running, the relationship is approximately constant (Abe, Fukuoka \& Horiuchi, 2015). Consequently, there is an intersection between the CoT-s relationship for walking and running; this is known as the energetically optimal transition speed (EOTS). However, the EOTS calculated in this way has been shown to be approximately 5-6 \% faster than the 'preferred' transition speed (PTS) at which humans make the walk-run transition (Hreljac, 1993; Rotstein et al., 2005; Tseh et al., 2002), so factors other than a minimization of the metabolic rate are needed to explain the trigger for the walk-run transition in human locomotion.

An abrupt increase in muscle activity of the dorsiflexor (tibialis anterior; TA) has been observed when participants walked at a speed close to the PTS (Hreljac, 1995; Hreljac et al., 2008; Prilutsky \& Gregor, 2001; Bartlett \& Kram, 2008; Malcolm et al., 2009; Shih et al., 2016), and our recent studies showed muscle activity of the TA decreased when participants switched from walking to running at the EOTS (Abe, Fukuoka \& Horiuchi, 2017; Abe et al., 2018). In association with a decrease in TA activity when switching the gait pattern, mean power frequency (MPF; Hz) of the TA became lower, suggesting that more Type 1 muscle fibers were recruited in the TA during running than walking at the EOTS. These findings suggest that muscle activity of the TA could be a trigger of the walk-run transition. Conversely, other reports have proposed that the medial head of gastrocnemius muscle (MG) of the plantar flexors, an antagonist of the TA, plays a key role in the walk-run transition (Farris \& Sawicki, 2012; Neptune \& Sasaki, 2005), because ankle force production by the MG was observed to decrease 
65 in proportion to the increase in walking speed. Thus, the trigger for the walk-run transition 66 remains under debate.

67 One approach to resolve this matter is to perform experiments using simulated reduced 68 gravity and/or body weight support (BWS) conditions. This reduces not only leg muscular 69 activity but also the required energy expenditure (EE) during walking (Grabowski, 2010; 70 McGowan, Kram \& Neptune, 2008; 2009; Pavei, Biancardi \& Minetti, 2015) and running 71 (Raffalt, Hovgaard-Hansen \& Jensen, 2013; Teunissen, Grabowski \& Kram, 2007). Because EE 72 is potentially reduced by the decreased plantar flexor activity (Hamner, Seth \& Delp 2010; 73 Neptune, Zajac \& Kautz, 2004), it would be expected that the muscular activity of the plantar 74 flexors would be lower in BWS than in normal walking (NW) conditions. In that case, the PTS 75 and/or EOTS would be faster in BWS than in NW conditions. Summarizing the results of the 76 above previous studies, we hypothesized that the PTS and/or EOTS would be faster in BWS 77 compared to NW. We also hypothesized that TA activities would decrease after the walk-run 78 transition under both conditions and that muscular activity of the plantar flexors would decrease 79 in BWS than in NW, while it remained constant regardless of walk-run transition. The purpose 80 of this study was to investigate the muscular activity and the motor unit recruitment patterns of 81 the dorsiflexor and plantar flexors before and after the walk-run transition under BWS and NW 82 conditions. 
83

84

85

86

87

88

89

90

91

92

93

94

95

96

97

98

99

100

101

102

103

104

105

106

107

108

109

110

111

112

113

114

\section{Materials \& Methods}

\section{Participants}

Thirteen young healthy males participated in the present study. Their physical characteristics are summarized in Table 1. In accordance with the Declaration of Helsinki, all participants were provided all information about the purposes, benefits, possible risks, and experimental protocols. A written informed consent was obtained from all participants. An ethical committee established in Kyushu Sangyo University approved all procedures of this study (H28-0001-1).

\section{Body weight support (BWS)}

BWS was established with a custom-made body suspension apparatus that lifted the participant's torso via an elastic rehabilitation harness (Fig. 1). This was installed around a motor-driven treadmill (Well Load 200E, Takei Scientific Instruments Co., Ltd., Niigata, Japan). For the BWS condition, we chose a $30 \%$ decrement in the participant's body weight (BW), equivalent to that used in some previous studies (Grabowski, 2010; McGowan, Kram \& Neptune, 2008; 2009; Raffalt, Hovgaard-Hansen \& Jensen, 2013; Teunissen, Grabowski \& Kram, 2007). A force transducer (TSA-110, Takei Scientific Instruments Co., Ltd., Niigata, Japan) was located between a control box and spring (with $30 \mathrm{~cm}$ free length and a spring constant of $5.7 \mathrm{~kg} \cdot \mathrm{cm}^{-1}$ ) to slide a rigid main frame (see supplemental video). Before the participant walked or ran, he stood stationary on the treadmill while wearing the harness, and $30 \%$ of his BW was lifted by the spring system. The set-up allowed normal leg swing.

\section{Protocols and determination of EOTS}

All participants continuously walked on a motor-driven treadmill at four walking speeds (1.33, $1.56,1.78$, and $\left.2.00 \mathrm{~m} \cdot \mathrm{s}^{-1}\right)$ on a level $( \pm 0 \%)$ gradient. After measuring the metabolic cost of walking, the participants took a sitting rest for 7-8 minutes. They started at three running speeds $\left(2.00,2.22\right.$, and $\left.2.44 \mathrm{~m} \cdot \mathrm{s}^{-1}\right)$ with 1 min standing rests between the running stages. Each walking and/or running speed was kept constant for $4 \mathrm{~min}$. At all stages, the participants chose their preferred step frequencies. They wore underwear, shirts, socks, and the same model of shoes in the appropriate sizes (Wave Wing, Mizuno, Japan).

Oxygen uptake $\left(\mathrm{VO}_{2} ; \mathrm{mL} \cdot \mathrm{kg}^{-1} \cdot \mathrm{s}^{-1}\right)$ and carbon dioxide output $\left(\mathrm{VCO}_{2} ; \mathrm{mL} \cdot \mathrm{kg}^{-1} \cdot \mathrm{s}^{-1}\right)$ were continuously measured with a computerized breath-by-breath system (AE-310S, Minato Ltd, Japan). Calibrated gas concentrations $\left(\mathrm{O}_{2} 15.22 \%, \mathrm{CO}_{2} 5.17 \%\right.$, and $\left.\mathrm{N}_{2} 79.61 \%\right)$ and room air 
115 were used for the calibration of the gas analyzer. An average $\mathrm{VO}_{2}$ and $\mathrm{VCO}_{2}$ for the final 2-min 116 at each speed was used to calculate the CoT values using a following equation (Brouwer, 1957). $\operatorname{CoT}\left(\mathrm{J} \cdot \mathrm{kg}^{-1} \cdot \mathrm{m}^{-1}\right)=\frac{4.186 \times(3.869 \times \mathrm{VO} 2+1.195 \times \mathrm{VCO} 2)}{s} \quad($ eq.1 $)$.

118 Where the $s$ is the walking or running speed. The CoT values were compared at each gait speed between both conditions. The relationship between the CoT values and walking speeds was approximated with a quadratic equation (Abe, Fukuoka \& Horiuchi, 2015):

$$
\operatorname{CoT}(s)=\mathrm{as} s^{2}+\mathrm{b} s+\mathrm{c} \quad(e q .2) .
$$

Where the coefficients $a, b$, and $c$ are determined by the least squares regression with data obtained from four walking speeds. The relationship between CoT values and running speeds was approximated using a linear equation (Abe, Fukuoka \& Horiuchi, 2015). The running CoT values was described as follows:

$$
\operatorname{CoT}(s)=\mathrm{p} s+\mathrm{q} \quad(e q .3) .
$$

Where the coefficients $\mathrm{p}$ and $\mathrm{q}$ are determined by the least squares regression with the CoT values from three running speeds. An extrapolation of the quadratic equation (eq.2) meets a linear regression line (eq.3), and then an intersection (EOTS) of these equations was obtained. This extrapolation has been normally used for determining the EOTS in other previous studies (Abe, Fukuoka \& Horiuchi, 2015; Hreljac, 1993; Minetti, Ardigò \& Saibene, 1994; Rotstein et al., 2005; Tseh et al., 2002). Thus, the EOTS is an extrapolated value when the eqs.2 and 3 are equal. Rearranging eqs.2 and 3:

$$
\mathrm{a} s^{2}+(\mathrm{b}-\mathrm{p}) s+(\mathrm{c}-\mathrm{q})=0 \quad(e q \cdot 4)
$$

A following formula gives two solutions of eq.4, thus, only the faster one is regarded as the EOTS (Abe, Fukuoka \& Horiuchi, 2015).

$$
\operatorname{EOTS}\left(\mathrm{m} \cdot \mathrm{s}^{-1}\right)=\frac{-(b-p) \pm \sqrt{(b-p)^{2}-4 a(c-q)}}{2 a} \quad(\text { eq.5). }
$$

\section{EMG measurements at the PTS}

The participant sat down on a chair to allow placement of the pre-amplified active surface EMG electrodes (BA-U410m, Nihon Santeku Co., LTD, Osaka) on tibialis anterior (TA), medial and lateral heads of gastrocnemius muscle (MG and LG), and soleus (SOL). Before electrode placement, the skin was shaved and wiped with alcohol for an exfoliation. The electrodes were secured using surgical tape to avoid disturbing the locomotor tasks. A foot sensor (PS-20KASF4, 
145 Kyowa Electronic Instruments Co., LTD., Tokyo) was inserted into a right shoe to count number 146 of steps, and its signal was amplified with a signal conditioner (CDV-700A, Kyowa Electronic 147 Instruments Co., LTD, Tokyo). This foot sensor also allowed us to visually detect what the 148 participant transitioned from walking to running, because the vertical force suddenly increased 149 when the participant started running. The vertical force itself should be ignored, because the foot 150 sensor is cushioned by the shoe sole and plantaris muscle. The participant was asked to start 151 walking at $1.11 \mathrm{~m} \cdot \mathrm{s}^{-1}$ for $1 \mathrm{~min}$ at a freely chosen step frequency. After this steady-state 152 walking, the treadmill speed was gradually increased at an acceleration of $0.00463 \mathrm{~m} \cdot \mathrm{sec}^{-2}(1$ $153 \mathrm{~km} \cdot \mathrm{h}^{-1}$ per minute) in a modified incremental ramp manner. The speed at which the walk-run 154 transition occurred was considered to be the PTS. The participant was asked to keep running for 1551 min after the walk-run transition. EMG was recorded throughout the whole ramp protocol. The 156 BWS condition was tested before the NW condition because it took several minutes to put on the

157 158 159

160

161

162 163 164

165

166

167

168

169

170

171

172

173

174

175

176 harness without disturbing the wired EMG electrodes and bio-amplifier attached to the participant's waist. Data for one participant was excluded from the analysis because of an artifact in the EMG. The analyzed time duration was set as $12 \mathrm{~s}$ before and after the walk-run transition; in practice, it was $11.972 \pm 1.562 \mathrm{~s}$, equivalent to $13.1 \pm 1.9$ steps when walking or $15.2 \pm 2.3$ steps when running.

The EMG signals were amplified with a gain of $\times 1000$ (BA 1104B, Digitex Lab Co., LTD, Tokyo). Sampling frequency was set at $2 \mathrm{kHz}$, and a band-pass filter (8-500 Hz) was applied for the EMG signals. All signals from each sensor were simultaneously recorded with software (MaP 1038 ver.7.4, Nihon Santeku Co., LTD, Osaka). Subsequently, a fast Fourier transform was applied to the stored EMG signals to obtain mean power frequency (MPF; Hz). MPF reflects motor unit recruitment pattern in the exercising muscles (De Luca, 1997; Wakeling, 2004). An alteration of the MPF values between walking and running allows us to evaluate muscle fiber recruitment pattern in each gait. The sum of the rectified EMG $(\mu \mathrm{V} \cdot \mathrm{sec})$ was normalized by measured time ( $\mathrm{sec}$ ) and number of steps to cancel the effects of step frequency (Abe, Fukuoka \& Horiuchi, 2017; Abe et al., 2018). The EMG values obtained were further normalized to those obtained at $1.11 \mathrm{~m} \cdot \mathrm{s}^{-1}$ under each condition.

\section{Statistical analysis}

Data were presented as mean $\pm \mathrm{SD}$. The CoT values were compared with two-way repeated measures analysis of variance $(\mathrm{ANOVA})$ within participants $(\mathrm{BW} \times$ speed $)$ using online software 
177 (ANOVA 4 Copyright (c) 2002 Kiriki Kenshi; https://www.hju.ac.jp/ kiriki/anova4/). Two-way 178 repeated measures of ANOVA was also applied for comparisons among the EOTS and PTS 179 obtained under both gravities. Each muscle activity and MPF were also compared in the same 180 way. The present study was exploratory in design, and the minimum detectable $F$ value was 1814.747 when using $G^{*}$ Power 3.1 (Faul et al., 2007) with an actual number of 13 participants. A 182 partial eta-squared value $\left(\eta^{2}\right)$ was also presented (Cohen, 1988). If a significant $F$ value was 183 obtained on the dependent variables, Ryan's post hoc test was applied to the appropriate data sets 184 to detect significant mean differences. Its statistical power has been reported to be equivalent to 185 Tukey's post hoc test (Ryan, 1960), and it can be used regardless of the data distribution (Ryan, 186 1960). Statistical significance was accepted at $p<0.05$. 
187

188

189

190

191

192

193

194

195

196

197

198

199

200

201

202

203

204

205

206

207

208

209

210

\section{Results}

Figure 2A shows the CoT-s relationships obtained under the two conditions. In walking, significantly lower CoT values were observed with BWS than with NW at speeds faster than $1.56 \mathrm{~m} \cdot \mathrm{s}^{-1}\left(F=16.019, p=0.002, \eta^{2}=0.178\right.$; Fig. 2A). In running, the CoT values were significantly lower with BWS than with NW at all speeds $(p<0.001)$. The mean EOTS was significantly faster with BWS than with NW $\left(2.304 \pm 0.141\right.$ vs. $2.031 \pm 0.086 \mathrm{~m} \cdot \mathrm{s}^{-1} ; F=32.326$, $p<0.001, \eta^{2}=0.327$; Fig. 2B). The same was the case with the PTS $(2.068 \pm 0.153$ vs. $1.932 \pm$ $0.075 \mathrm{~m} \cdot \mathrm{s}^{-1} ; F=43.209, p<0.001, \eta^{2}=0.331$; Fig. 2B). A post hoc test revealed that the PTS was significantly slower than the EOTS under both conditions $(F=7.865, p=0.010$; Fig. 2B).

Comparisons of activities of the dorsiflexor (TA) and the combined plantar flexor activities (the average for MG, LG, and SOL) are shown in the upper panels of Figure 3. TA activity significantly decreased after the walk-run transition under both conditions $(F=12.896, p=$ $0.004, \eta^{2}=0.183$; Fig. 3A). Muscle activities of the combined plantar flexors also decreased after the walk-run transition with both BWS $\left(F=57.091, p<0.001, \eta^{2}=0.111\right.$; Fig. 3B) and NW $\left(F=5.177, p=0.026, \eta^{2}=0.019\right.$; Fig. 3B). The lower panels of Figure 3 show each muscle activity of the synergic plantar flexors; only SOL activity became significantly lower after the walk-run transition under both conditions $\left(F=33.539, p<0.001, \eta^{2}=0.203\right.$; Fig. 3E).

Figure 4 shows comparisons of the MPF values for each muscle. The MPF of the TA was significantly lower after the walk-run transition under both conditions $\left(F=7.484, p=0.019, \eta^{2}=\right.$ 0.028; Fig. 4A), whereas the MPF of the MG was significantly higher after the walk-run transition only with the BWS $\left(F=10.516, p=0.004, \eta^{2}=0.067\right.$; Fig. 4B). No significant differences were observed for the LG $(F=0.125, p=0.730$, Fig. 4C). The SOL showed significantly lower MPF values after the walk-run transition under both conditions $(F=22.355$, $p<0.001, \eta^{2}=0.057$; Fig. 4D). 


\section{Discussion}

212 One of the major findings of this study was that the walking CoT values were significantly lower 213 with BWS than with NW at speeds faster than $1.56 \mathrm{~m} \cdot \mathrm{s}^{-1}$ (Fig. 2A). The running CoT values 214 were also lower with BWS than with NW at all speed measured (Fig. 2A); this was consistent 215 with the results of previous studies (Raffalt, Hovgaard-Hansen \& Jensen, 2013; Teunissen, 216 Grabowski \& Kram, 2007; Pavei, Biancardi \& Minetti, 2015; Pavei \& Minetti, 2016;

217 Grabowski, Farley \& Kram, 2005; Grabowski \& Kram, 2008). These results also demonstrated 218 that the EOTS was faster in BWS than in NW (Fig. 2B). Although it has been proposed that the 219 walk-run transition minimizes whole-body EE (Cavagna \& Kaneko, 1977; Minetti, Ardigò \& 220 Saibene, 1994), other previous studies have found that the PTS was 5-6\% slower than the EOTS 221 in humans (Hreljac, 1993; Rotstein et al., 2005; Tseh et al., 2002), suggesting that the other 222 factors may be related to the walk-run transition. The results of the present study were consistent 223 with those of the latter studies (Hreljac, 1993; Rotstein et al., 2005; Tseh et al., 2002), with the 224 PTS being significantly slower than the EOTS by $5.2 \%$ under the NW condition and 11.4\% 225 under the BWS condition (Fig. 2B). These results indicate that the walk-run transition is not 226 triggered exclusively to minimize whole-body EE.

227 In support of our first hypothesis, TA activity and the associated MPF decreased when the 228 gait pattern was switched from walking to running under both conditions (Figs. 3A and 4A). 229 These results were equivalent to those of our recent studies that measured TA activity during 230 walking and running at the EOTS (Abe, Fukuoka \& Horiuchi, 2017; Abe et al., 2018), which 231 suggested that the motor unit recruitment pattern of the TA shifted more toward Type I (slow 232 twitch) fibers rather than Type II (fast twitch) fibers after the walk-run transition. Several studies 233 have also reported that an abrupt increase in TA activity associated with the walk-run transition 234 (Hreljac, 1995; Hreljac et al., 2008; Bartlett \& Kram, 2008; Malcolm et al., 2009; Shih et al., 235 2016). However, a careful consideration is necessary, because the TA does not contribute to 236 producing mechanical power for forward acceleration. It is the ankle plantar flexors (MG, LG, 237 and SOL), that play an essential role in forward acceleration during walking (Francis et al., 238 2013; Franz \& Thelen, 2016; Gottschall \& Kram, 2003) and running (Hamner, Seth \& Delp, 239 2010). Indeed, the MG might be a potential trigger for the walk-run transition, because its power 240 production has been shown to decrease when walking faster (Farris \& Sawicki, 2012; Neptune \& 241 Sasaki, 2005). However, these previous results were based on simulated calculations; functional 242 differences in other synergic ankle plantar flexors remain to be elucidated during human 
243 locomotion. We found that the combined activities of the plantar flexors (the average of $\mathrm{MG}$, 244 LG, and SOL activities) were significantly lower during running than walking under both 245 conditions (Figs. 3A and 3B). These results clearly suggest that the plantar flexors also 246 contribute to triggering the walk-run transition.

247 Considering muscle activity between before and after the walk-run transition, LG activity 248 decreased significantly after the walk-run transition only under the BWS condition (Fig. 3C), and 249 the MPF of both MG and LG was not significantly different between before and after the walk250 run transition, except for the MPF of the MG under the BWS (Figs. 4B and 4C). These results 251 were partly consistent with the results of our recent studies (Abe, Fukuoka \& Horiuchi, 2017; 252 Abe et al., 2018). The inconsistency in MG and LG activities can be explained by SOL activity. 253 The functional role of the SOL has not been as well investigated as that of the MG and LG 254 complex. In fact, the gastrocnemius muscles and SOL are anatomically independent. The 255 anatomical volume of the SOL is much larger than that of the MG and LG complex, but the SOL 256 exerts more mechanical power in the upward direction (Franz \& Thelen, 2016). Thus, the SOL 257 should contribute to supporting the body during walking. Another study reported that a 258 difference of the functional role between the gastrocnemius muscles and SOL mainly appeared 259 in the late stance phase (Zajac, Neptune \& Kautz, 2003). The SOL in association with the MG 260 and LG complex support the body in the early and middle stance phases (Neptune, Zajac \& 261 Kautz, 2001), but the push-off is mainly executed by the SOL only in the late stance phase 262 (Zajac, Neptune \& Kautz, 2003). These previous studies suggest that the SOL executes multi263 tasks in one gait cycle: forward acceleration of the trunk in the late stance phase and body 264 support in the early and middle stance phases.

265 One of the major limitations of our experimental approach was that we could not quantify in 266 vivo Achilles tendon behavior. The ankle torque produced by the plantar flexors increases as 267 walking speed increases. However, the change in length of the SOL and its tendon complex has 268 been observed to decrease drastically during walking around the PTS (Lai et al., 2015), 269 suggesting an extreme increase in the shortening length of the SOL itself in a gait cycle. This 270 potentially increases the force production by the SOL to compensate for the decreased length 271 change of the SOL and tendon complex in a gait cycle. After the walk-run transition, length 272 change of the SOL abruptly increased (Lai et al., 2015), indicating that the SOL force production 273 and related activations can be reduced. This finding was supported by our result for changes in 274 SOL activity under both conditions (Fig. 3E). In addition, the MPF of the SOL decreased at the 
275 walk-run transition (Fig. 4D), indicating that the SOL plays a role not only in supporting the 276 body but also in triggering the walk-run transition. Given these complex observations, the ankle 277 plantar flexors may all contribute to the required forces, in association with changes to each 278 motor unit recruitment pattern, to avoid the early onset of localized muscle fatigue.

279 Methodological considerations are necessary, because we found a faster PTS in BWS than that in 280 NW, which contradicted numerous previous results (e.g. Kram, Domingo \& Ferris, 1999). This 281 conflicting result could be attributed to a difference of the used apparatus. The BWS apparatus 282 used in some previous studies retained much larger stretch in the elastic component compared to 283 our apparatus (Kram, Domingo \& Ferris, 1999; Pavei, Biancardi \& Minetti, 2015; Pavei \& 284 Minetti, 2016), and small vertical movements of the participants using our apparatus could result 285 in fluctuations of the vertical force. Figure 5 shows a representative example of fluctuations of 286 the vertical force (weight) during both gaits in the BWS condition. The baseline represents $70 \%$ 287 of this participant's BW (30\% reduction of his BW from $63.2 \mathrm{~kg}$ to $43.8 \mathrm{~kg}$ ), so the suspended 288 weight is $18.8 \mathrm{~kg}$. As the fluctuation of the vertical force was about $\pm 4 \mathrm{~kg}$ during running, it was 289 fluctuated between $24 \% \mathrm{BW}$ and $36 \% \mathrm{BW}$ during running. During walking, the fluctuation of the 290 vertical force was within $\pm 2 \mathrm{~kg}$, indicating that it was fluctuated between $27 \% \mathrm{BW}$ and $33 \% \mathrm{BW}$ 291 during walking. These fluctuations of the vertical force are not negligible, so we used the term 292 body weight support (BWS) rather than simulated reduced gravity. In the previous studies, the 293 PTS became slower as a function of reduced body weight level (Kram, Domingo \& Ferris, 1999; 294 Pavei, Biancardi \& Minetti, 2015; Pavei \& Minetti, 2016), which can be predicted from the 295 Froude number (Alexander, 1989). The spring of our apparatus is much shorter (only $30 \mathrm{~cm}$ ) than 296 that of previously used apparatus due to a limitation of the room height. Thus, our spring-like 297 apparatus yielded relatively large fluctuations of the vertical force. This intrinsic characteristic of 298 299 300 our apparatus could reduce the mechanical power output for the vertical direction, which should be related to the decreased whole-body EE during running and walking at faster speeds (Fig. 2B), resulting in a faster PTS in BWS than in NW.

There had been a debate whether the spectral properties of the surface EMG provide information about motor unit recruitment strategies and muscle fiber type (von Tscharner \& Nigg, 2008; Farina, 2008). In recent years, many articles provided the motor unit activity using 305 power spectral properties (MPF) of the surface EMG during dynamic exercise in vivo (De Luca 306 et al., 2015; Kallio et al., 2013; von Tscharner et al., 2018) along with the Henneman's size principle (Henneman, Somjen \& Carpenter, 1965). To the best of our knowledge, Henneman's 
307 size principle cannot be applied during instantaneous heavy power output (Moritani, Oddsson \& 308 Thorstensson, 1990) and/or instantaneous eccentric contraction (Smith et al., 1980; Nardone \& 309 Schieppati, 1988; Kallio et al., 2013). Our experimental condition partially met such specific 310 conditions, suggesting that alterations of the MPF values before and after the walk-run transition 311 reflected a shift toward more Type 1 motor unit recruitment. That is, the walk-run transition is 312 associated with greater slow twitch fiber recruitment of the TA and SOL, perhaps to avoid early 313 onset of localized muscle fatigue.

314

\section{Conclusions}

316 The PTS was significantly slower than the EOTS under both conditions, indicating that the walk317 run gait transition is not only triggered by the minimization of whole-body EE. In the BWS 318 condition, both PTS and EOTS were faster due to the potential reduction of muscular activity in 319 both the dorsiflexor (TA) and the plantar flexors, particularly in the SOL. Reductions in the 320 activity of these muscles in association with changes to the motor unit recruitment patterns make 321 a considerable contribution to the walk-run transition in human locomotion. 


\section{Acknowledgements}

323 We specially thank to Mr. Akinobu Sakamoto, Mr. Tomokazu Iwatani, Mr. Hiromichi Ikegami, 324 Mr. Takeshi Saito, Mr. Masaru Hashimura, and Mr. Shizuo Takatoh (Takei Scientific

325 Instruments Co., Ltd.) for customizing both body suspension apparatus and treadmill. We also

326 thank to Hideki Kaneko and Shouichi Matsuyama (Nihon Santeku Co., Ltd.) for their continuous

327 technical assistance of the EMG recording and analysis. 
328

329

330

331

332

333

334

335

336

337

338

339

340

341

342

343

344

345

346

347

348

349

350

351

352

353

354

355

356 Farris DJ, Sawicki GS. 2012. Human medial gastrocnemius force-velocity behavior shifts with

357 locomotion speed and gait. Proceedings of the National Academy of Sciences of the United

358 States of America 109:997-982.

\section{References}

Abe D, Fukuoka Y, Horiuchi M. 2015. Economical speed and energetically optimal transition speed evaluated by gross and net oxygen cost of transport at different gradients. PLoS One 10:e0138154.

Abe D, Fukuoka Y, Maeda T, Horiuchi M. 2018. Energy cost and lower leg muscle activities during erect bipedal locomotion under hyperoxia. Journal of Physiological Anthropology 37:18. Abe D, Fukuoka Y, Horiuchi M. 2017. Muscle activities during walking and running at energetically optimal transition speed under normobaric hypoxia on gradient slopes. PLoS One 12:e0173816.

Alexander RM. 1989. Optimization and gaits in the locomotion of vertebrates. Physiological Review 69:1199-1227.

Bartlett JL, Kram R. 2008. Changing the demand on specific muscle groups affects the walkrun transition speed. Journal of Experimental Biology 211:1281-1288.

Brouwer E. 1957. On simple formulae for calculating the heat expenditure and the quantities of carbohydrate and fat oxidized in metabolism of men and animals, from gaseous exchange (Oxygen intake and carbonic acid output) and urine-N. Acta physiologica et pharmacologica Neerlandica 6:795-802.

Cavagna GA, Kaneko M. 1977. Mechanical work and efficiency in level walking and running. Journal of Physiology 268:467-481.

Cohen J. 1988. Statistical power analysis for the behavioral sciences. 2nd edition. Hillsdale: L. Erlbaum Associates. pp.283.

De Luca CJ. 1997. The use of surface electromyography in biomechanics. Journal of Applied Biomechanics 13:135-163.

De Luca CJ, Chang SS, Roy SH, Kline JC, Nawab SH. 2015. Decomposition of surface EMG signals from cyclic dynamic contractions. Journal of Neurophysiology 113:1941-1951.

Farina D. 2008. Point: Counterpoint: Spectral properties of the surface EMG do not provide information about motor unit recruitment and muscle fiber type. Journal of Applied Physiology 105:1683. 
359 Faul F, Erdfelder E, Lang A-G, Buchner A. 2007. G*Power 3: a flexible statistical power 360 analysis program for the social, behavioral, and biomedical sciences. Behavior Research 361 Methods 39:175-191.

362 Francis CA, Lenz AL, Lenhart RL, Thelen DG. 2013. The modulation of forward propulsion, 363 vertical support, and center of pressure by the plantarflexors during human walking. Gait \& 364 Posture 38:993-997.

365 Franz JR, Thelen DG. 2016. Imaging and simulation of Achilles tendon dynamics: Implications 366 for walking performance in the elderly. Journal of Biomechanics 49:1403-1410.

367 Gottschall JS, Kram R. 2003. Energy cost and muscular activity required for propulsion during 368 walking. Journal of Applied Physiology 94:1766-1772.

369 Grabowski A, Farley CT, Kram R. 2005. Independent metabolic costs of supporting body 370 weight and accelerating body mass during walking. Journal of Applied Physiology 98:579-583.

371 Grabowski AM, Kram R. 2008. Effects of velocity and weight support on ground reaction 372 forces and metabolic power during running. Journal of Applied Biomechanics 24:288-297.

373 Grabowski AM. 2010. Metabolic and biomechanical effects of velocity and weight support 374 using a lower-body positive pressure device during walking. Archives of Physical Medicine and 375 Rehabilitation 91:951-957.

376 Hamner SR, Seth A, Delp SL. 2010. Muscle contributions to propulsion and support during 377 running. Journal of Biomechanics 43:2709-2716.

378 Henneman E, Somjen G, Carpenter DO. 1965. Functional significance of cell size in spinal 379 motoneurons. Journal of Neurophysiology 28:560-580.

380 Hreljac A. 1993. Preferred and energetically optimal gait transition speeds in human 381 locomotion. Medicine \& Science in Sports \& Exercise 25:1158-1162.

382 Hreljac A. 1995. Determinants of the gait transition speed during human locomotion: kinematic 383 factors. Journal of Biomechanics 28:669-677.

384 Hreljac A, Imamura RT, Escamilla RF, Edwards WB, MacLeod T. 2008. The relationship 385 between joint kinetic factors and the walk-run gait transition speed during human locomotion. 386 Journal of Applied Biomechanics 24:149-157.

387 Kallio J, Søgaard K, Avela J, Komi PV, Selänne H, Linnamo V. 2013. Motor unit firing 388 behaviour of soleus muscle in isometric and dynamic contractions. PLoS One 8:e53425. 389 Kram R, Domingo A, Ferris DP. 1997. Effect of reduced gravity on the preferred walk-run 390 transition speed. Journal of Experimental Biology 200:821-826. 
391 Lai A, Lichtwark GA, Schache AG, Lin YC, Brown NA, Pandy MG. 2015. In vivo behavior 392 of the human soleus muscle with increasing walking and running speeds. Journal of Applied 393 Physiology 118:1266-1275.

394 Malatesta D, Simar D, Dauvilliers Y, Candau R, Borrani F, Prefaut C, Caillaud C. 2003. 395 Energy cost of walking and gait instability in healthy 65- and 80-yr-olds. Journal of Applied 396 Physiology 95:2248-2256.

397 Malcolm P, Segers V, Van Caekenberghe I, De Clercq D. 2009. Experimental study on the 398 influence of the $\mathrm{m}$. tibialis anterior on the walk-to-run transition by means of powered ankle-foot 399 exoskeleton. Gait \& Posture 29:6-10.

400 Margaria R. 1938. Sulla fisiologia e specialmente sul consume energetico della marcia e della 401 corsa a varia velocita` ed inclinazione del terreno. Atti della Accademia Nazionale del Lincei $402 \quad 7: 299-368$.

403 McGowan CP, Kram R, Neptune RR. 2008. Independent effects of weight and mass on plantar 404 flexor activity during walking: implications for their contributions to body support and forward 405 propulsion. Journal of Applied Physiology 105:486-494.

406 McGowan CP, Kram R, Neptune RR. 2009. Modulation of leg muscle function in response to 407 altered demand for body support and forward propulsion during walking. Journal of 408 Biomechanics 42:850-856.

409 Minetti AE, Ardigò LP, Saibene F. 1994. The transition between walking and running in 410 humans: metabolic and mechanical aspects at different gradients. Acta Physiologica 411 Scandinavica 150:315-323.

412 Moritani T, Oddsson L, Thorstensson A. 1990. Differences in modulation of the 413 gastrocnemius and soleus H-reflexes during hopping in man. Acta Physiologica Scandinavica $414 \quad 138: 575-576$.

415 Nardone A, Schieppati M. 1988. Shift of activity from slow to fast muscle during voluntary 416 lengthening contractions of the triceps surae muscles in humans. Journal of Physiology 395:363417381.

418 Neptune RR, Kautz SA, Zajac FE. 2001. Contributions of the individual ankle plantar flexors 419 to support, forward progression and swing initiation during walking. Journal of Biomechanics $42034: 1387-1398$. 
421 Neptune RR, Zajac FE, Kautz SA. 2004. Muscle mechanical work requirements during normal

422 walking: the energetic cost of raising the body's center-of-mass is significant. Journal of

423 Biomechanics 37:817-825.

424 Neptune RR, Sasaki K. 2005. Ankle plantar flexor force production is an important determinant 425 of the preferred walk-to-run transition speed. Journal of Experimental Biology 208:799-808.

426 Pavei G, Biancardi CM, Minetti AE. 2015. Skipping vs. running as the bipedal gait of choice

427 in hypogravity. Journal of Applied Physiology 119:93-100.

428 Pavei G, Minetti AE. 2016. Hopping locomotion at different gravity: metabolism and 429 mechanics in humans. Journal of Applied Physiology 120:1223-1229.

430 Prilutsky BI, Gregor RJ. 2001. Swing- and support-related muscle actions differentially trigger 431 human walk-run and run-walk transitions. Journal of Experimental Biology 204:2277-2287.

432 Raffalt PC, Hovgaard-Hansen L, Jensen BR. 2013. Running on a lower-body positive 433 pressure treadmill: VO2max, respiratory response, and vertical ground reaction force. Research 434 Quarterly for Exercise and Sport 84:213-222.

435 Rotstein A, Inbar O, Berginsky T, Meckel Y. 2005. Preferred transition speed between 436 walking and running: effects of training status. Medicine \& Science in Sports \& Exercise $437 \quad 37: 1864-1170$.

438 Ryan TA. 1960. Significance tests for multiple comparison of proportions, variances, and other 439 statistics. Psychological Bulletin 57:318-328.

440 Shih Y, Chen YC, Lee YS, Chan MS, Shiang TY. 2016. Walking beyond preferred transition 441 speed increases muscle activations with a shift from inverted pendulum to spring mass model in 442 lower extremity. Gait \& Posture 46:5-10.

443 Smith JL, Betts B, Edgerton VR, Zernicke RF. 1980. Rapid ankle extension during paw 444 shakes: selective recruitment of fast ankle extensors. Journal of Neurophysiology 43:612-620.

445 Teunissen LP, Grabowski A, Kram R. 2007. Effects of independently altering body weight 446 and body mass on the metabolic cost of running. Journal of Experimental Biology 210:44184474427.

448 von Tscharner V, Nigg BM. 2008. Point: spectral properties of the surface EMG can 449 characterize/do not provide information about motor unit recruitment strategies and muscle fiber 450 type. Journal of Applied Physiology 105:1671-1673. 
451 von Tscharner V, Ullrich M, Mohr M, Comaduran Marquez D, Nigg BM. 2018. A wavelet

452 based time frequency analysis of electromyograms to group steps of runners into clusters that

453 contain similar muscle activation patterns. PLoS One 13:e0195125.

454 Tseh W, Bennett J, Caputo JL, Morgan DW. 2002. Comparison between preferred and

455 energetically optimal transition speeds in adolescents. European Journal of Applied Physiology

456 88:117-121.

457 Wakeling JM. 2004. Motor units are recruited in a task-dependent fashion during locomotion.

458 Journal of Experimental Biology 207:3883-3890.

459 Zajac FE, Neptune RR, Kautz SA. 2003. Biomechanics and muscle coordination of human

460 walking: part II: lessons from dynamical simulations and clinical implications. Gait \& Posture $461 \quad 17: 1-17$. 
462

463

464

465

466

467

468

469

470

471

472

473

474

475

476

477

478

479

480

481

482

483

484

485

486

487

488

489

490

491

492

493

\section{Table and Figure Legends}

Table 1. Physical characteristics of each participant.

BW; body weight, BF; percent of body fat, and BMI; body mass index, respectively.

\section{Fig. 1. Body weight support apparatus.}

A schematic illustration of the participants walking with a custom-made body weight support (BWS) apparatus. This setting ensured that the BWS apparatus applied purely vertical force without disturbing leg swing motion.

\section{Fig. 2. Comparisons of CoT values under normal walking (NW) and body weight support} (BWS) conditions.

(A) Cost of transport (CoT) as a function of gait speed under NW (black solid and open squares) and BWS (blue solid and open circles). Solid lines are the approximations using actually observed CoT values. Dotted lines are the extrapolations. (B) Comparisons of energetically optimal transition speed (EOTS) and preferred transition speed (PTS) under NW (black-white bars) and BWS (blue-green bars). Values are mean \pm SD. ${ }^{*} p<0.05,{ }^{\#} p<0.01$, and ${ }^{\dagger} p<0.001$.

\section{Fig. 3. Comparisons of muscle activities.}

Comparisons of dorsiflexor (A; tibialis anterior, TA) and average of a complex of the entire plantar flexors (B). Lower panels presented each muscle activity of the gastrocnemius medialis $(\mathrm{C} ; \mathrm{MG})$, gastrocnemius lateralis (D; LG), and soleus $(\mathrm{E} ; \mathrm{SOL})$ before and after the walk-run transition under the NW and BWS. Values are mean \pm SD. ${ }^{*} p<0.05$ and ${ }^{\#} p<0.01$.

\section{Fig. 4. Comparisons of mean power frequency (MPF).}

Comparisons of MPF in the lower leg extremities before and after walk-run transition under NW and BWS. (A) TA: tibialis anterior; (B) MG: gastrocnemius medialis; (C) LG: gastrocnemius lateralis; and (D) SOL: soleus, respectively. Values are mean \pm SD. ${ }^{*} p<0.05$ and ${ }^{\#} p<0.01$.

\section{Fig. 5. Time-vertical force relationship using body weight support (BWS) apparatus.} A representative example of fluctuations of the vertical force during walking (upper panels) and running (lower panels) at each speed in the BWS condition. BW means body weight. 


\section{Table $\mathbf{1}$ (on next page)}

Table 1. Physical characteristics of each participant.

BW; body weight, BF; percent of body fat, and BMI; body mass index, respectively. 
Table 1. Physical characteristics of each participant.

\begin{tabular}{cccccc}
\hline & Age & Stature & BW & BF & BMI \\
\cline { 2 - 6 } Participant & $(\mathrm{yrs})$ & $(\mathrm{m})$ & $(\mathrm{kg})$ & $(\%)$ & $\begin{array}{c}\left(\mathrm{kg} \cdot \mathrm{m}^{-}\right. \\
2\end{array}$ \\
\hline A & 19.6 & 1.696 & 72.0 & 19.8 & 25.0 \\
B & 21.1 & 1.700 & 66.4 & 19.8 & 23.0 \\
C & 21.7 & 1.638 & 56.5 & 16.2 & 21.1 \\
D & 19.8 & 1.703 & 54.2 & 10.6 & 18.7 \\
E & 19.8 & 1.635 & 62.6 & 20.2 & 23.4 \\
F & 20.0 & 1.650 & 70.0 & 25.1 & 25.7 \\
G & 21.2 & 1.665 & 66.5 & 16.5 & 24.0 \\
H & 19.5 & 1.684 & 56.9 & 13.0 & 20.1 \\
I & 19.9 & 1.740 & 81.0 & 24.3 & 26.8 \\
J & 19.3 & 1.634 & 56.7 & 17.6 & 21.2 \\
K & 20.6 & 1.736 & 49.2 & 10.4 & 16.3 \\
L & 19.9 & 1.746 & 55.8 & 13.6 & 18.3 \\
M & 21.3 & 1.620 & 56.4 & 17.6 & 21.5 \\
\hline Average & 20.3 & 1.681 & 61.9 & 17.3 & 21.9 \\
SD & 0.8 & 0.044 & 8.9 & 4.6 & 3.1 \\
\hline BW body & BF & & & & \\
\hline
\end{tabular}

BW; body weight, BF; percent of body fat, and BMI; body mass index, respectively. 


\section{Figure 1}

Figure 1. Body weight support apparatus.

A schematic illustration of the participants walking with a custom-made body weight support (BWS) apparatus. This setting ensured that the BWS apparatus applied purely vertical force without disturbing leg swing motion. 


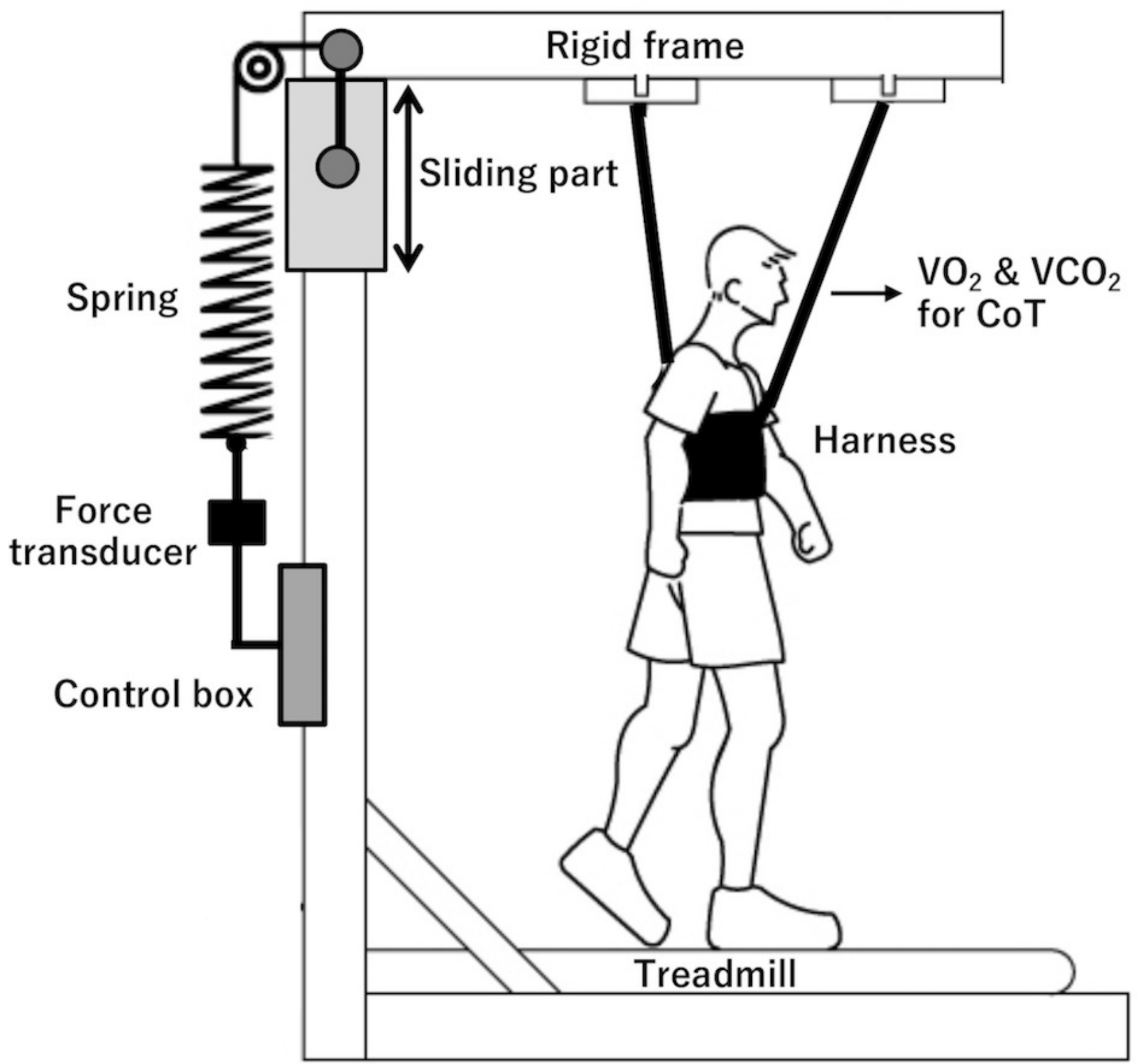




\section{Figure 2}

Comparisons of CoT values under normal walking (NW) and body weight support (BWS) conditions.

(A) Cost of transport (COT) as a function of gait speed under NW (black solid and open squares) and BWS (blue solid and open circles). Solid lines are the approximations using actually observed CoT values. Dotted lines are the extrapolations. (B) Comparisons of energetically optimal transition speed (EOTS) and preferred transition speed (PTS) under NW (black-white bars) and BWS (blue-green bars). Values are mean \pm SD. ${ }^{*} p<0.05,{ }^{*} p<0.01$, and ${ }^{\dagger} p<0.001$ 

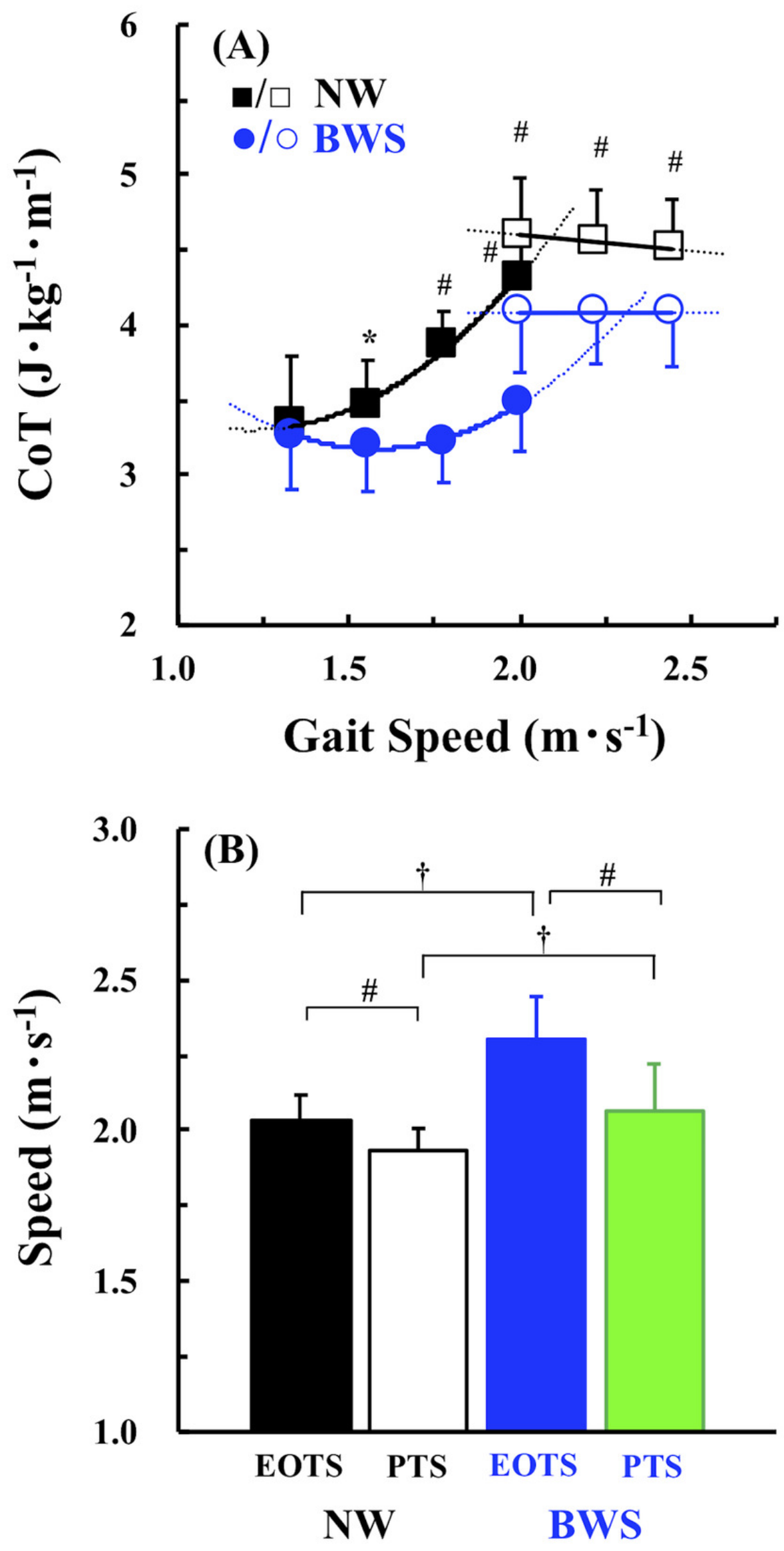


\section{Figure 3}

Figure 3. Comparisons of muscle activities.

Comparisons of dorsiflexor ( $\mathrm{A}$; tibialis anterior, TA) and average of a complex of the entire plantar flexors (B). Lower panels presented each muscle activity of the gastrocnemius medialis (C; MG), gastrocnemius lateralis (D; LG), and soleus (E; SOL) before and after the walk-run transition under the NW and BWS. Values are mean \pm SD. ${ }^{*} p<0.05$ and ${ }^{\#} p<0.01$.

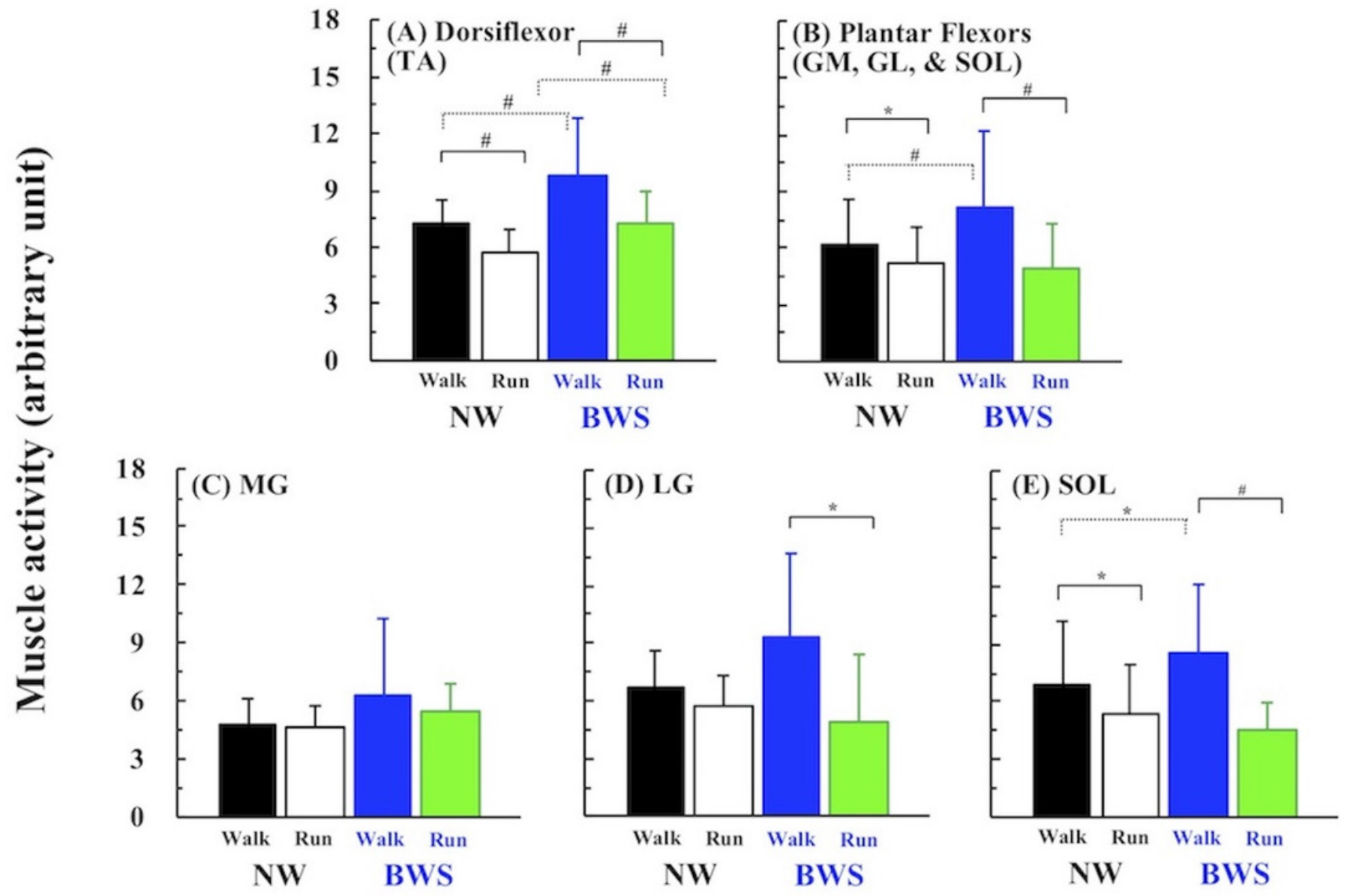




\section{Figure 4}

Figure 4. Comparisons of mean power frequency (MPF).

Comparisons of MPF in the lower leg extremities before and after gait transition under NW and BWS. (A) TA: tibialis anterior; (B) GM: gastrocnemius medialis; (C) GL: gastrocnemius lateralis; and (D) SOL: soleus, respectively. Values are mean \pm SD. ${ }^{*} p<0.05$ and ${ }^{*} p<0.01$. 

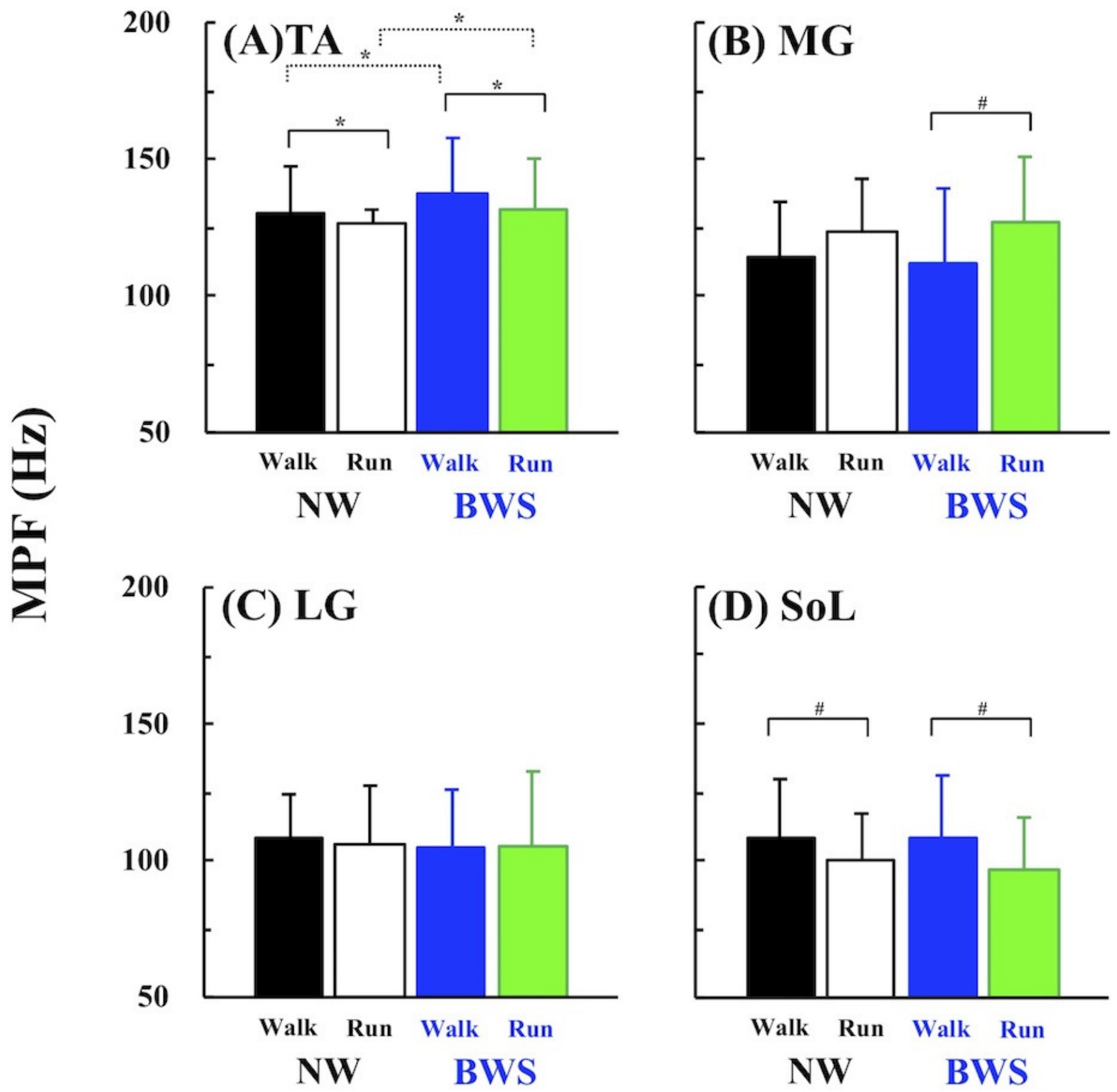
Figure 5

Figure 5. Time-vertical force relationship using body weight support (BWS) apparatus.

A representative example of fluctuations of the vertical force during walking (upper panels) and running (lower panels) at each speed in the BWS condition. BW means body weight.

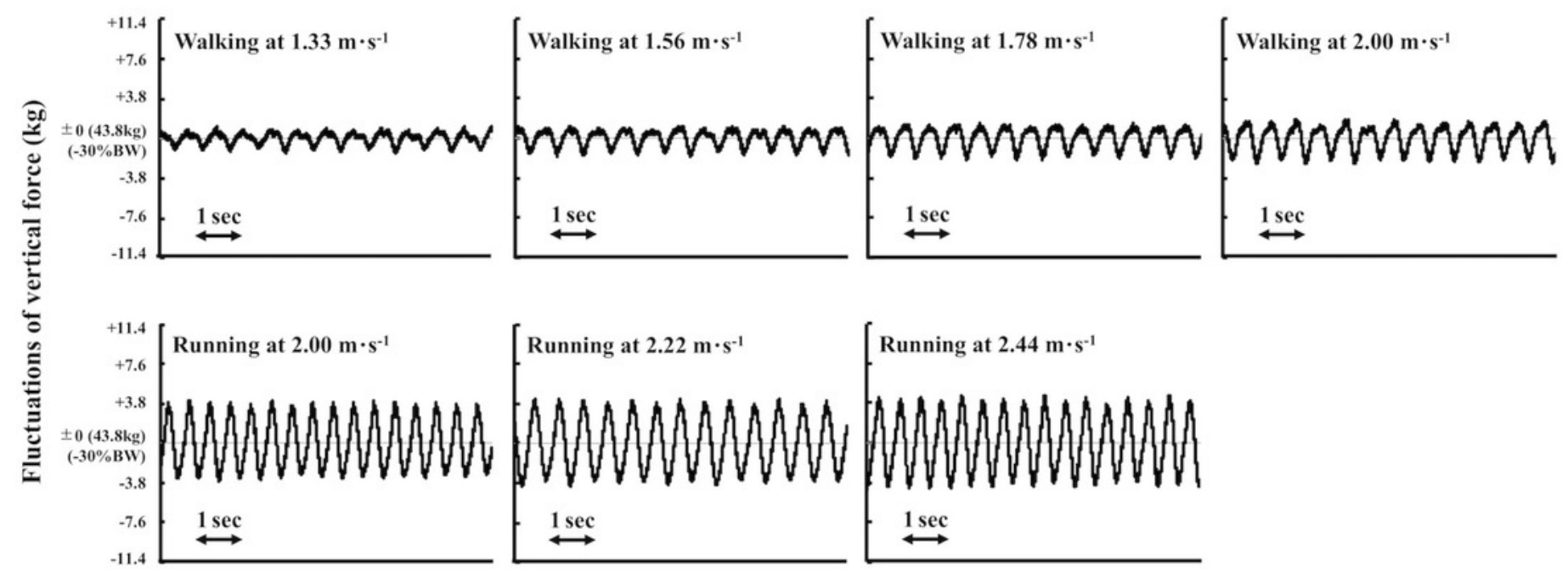

